\title{
Effect of zinc and boron application on productivity, quality and nutrient uptake of fieldpea (Pisum sativum L.) grown in calcareous soils
}

\author{
M. A. Quddus ${ }^{1}$, M. A. Hossain ${ }^{2}$, H. M. Naser ${ }^{2}$, B. Anwar ${ }^{3}$, S. Aktar ${ }^{4}$ and M. Nazimuddin ${ }^{5}$ \\ ${ }^{1}$ Soil and Water Management Section, Horticulture Research Centre, Bangladesh Agricultural Research Institute, \\ Gazipur, Bangladesh. \\ ${ }^{2}$ Soil Science Division, Bangladesh Agricultural Research Institute, Gazipur, Bangladesh. \\ ${ }^{3}$ Regional Agricultural Research Station, Bangladesh Agricultural Research Institute, Jashore, Bangladesh. \\ ${ }^{4}$ Pulses Research Sub-Station, Bangladesh Agricultural Research Institute, Gazipur, Bangladesh. \\ ${ }^{5}$ Olericulture Division, Horticulture Research Centre, Bangladesh Agricultural Research Institute, Gazipur, Bangladesh. \\ ${ }^{*}$ Corresponding author. Email: quddus06@yahoo.com \\ Copyright ( 2018 Quddus et al. This article remains permanently open access under the terms of the Creative Commons Attribution License 4.0, \\ which permits unrestricted use, distribution, and reproduction in any medium, provided the original work is properly cited.
}

Received 20th November, 2018; Accepted 12th December, 2018

\begin{abstract}
An experiment was conducted for two consecutive years (2014-15 and 2015-16) at Regional Pulses Research Station, Madaripur and Regional Agricultural Research Station, Jashore, Bangladesh during Rabi (winter) season to evaluate the effect of Zinc (Zn) and Boron (B) on productivity, nodulation, nutrient uptake and quality of fieldpea (Pisum sativum L.) and how these elements can help to manage soil fertility. There were sixteen treatment combinations comprising four levels of $\mathrm{Zn}\left(0,1.0,2.0\right.$ and $\left.3.0 \mathrm{~kg} \mathrm{ha}^{-1}\right)$ and four levels of Boron $\left(0,1.0,1.5\right.$ and $\left.2.0 \mathrm{~kg} \mathrm{ha}^{-1}\right)$ along with a blanket dose of fertilizers of $\mathrm{N}, \mathrm{P}, \mathrm{K}$ and $\mathrm{S}$ at $12,22,30$ and $10 \mathrm{~kg} \mathrm{ha}^{-1}$, respectively used in all combination. The experiment was laid out in a split-plot design with three replications. Results showed that the treatment combination of $\mathrm{Zn}_{3.0} \mathrm{~B}_{2.0}$ produced significantly higher seed yield followed by treatment combination of $Z n_{3.0} B_{1.5}$. The lowest seed yield was found in control $\left(\mathrm{Zn}_{0} \mathrm{~B}_{0}\right)$ combination. Treatment combination of $\mathrm{Zn}$ at $3 \mathrm{~kg} \mathrm{ha}^{-1}$ and $\mathrm{B}$ at $2 \mathrm{~kg} \mathrm{ha}^{-1}$ resulted in higher yield increment of $76.3 \%$ at Madaripur and $64.3 \%$ at Jashore over the control treatment $\left(Z n_{0} B_{0}\right)$. Root nodulation and seed protein content was found highest in $\mathrm{Zn}_{3.0} \mathrm{~B}_{2.0}$ treatment at both the locations. Zinc and Boron uptake by the fieldpea was also significantly affected by the added of $\mathrm{Zn}$ and $\mathrm{B}$ fertilizer. The combine application of $\mathrm{Zn}$ and $\mathrm{B}$ was superior to single application. The treatment combination of $Z n_{3.0} B_{2.0}$ followed by $Z n_{3.0} B_{1.5}$ showed positive results in improving soil organic matter, N, P, S, Zn and B content in soil for both locations. Hence, the results recommended that combine application of $\mathrm{Zn}$ and $\mathrm{B}$ either at of 3 and $2 \mathrm{~kg} \mathrm{ha}^{-1}$ or at of 3 and $1.5 \mathrm{~kg} \mathrm{ha}^{-1}$, respectively along with blanket fertilizers of $\mathrm{N}_{12} \mathrm{P}_{22} \mathrm{~K}_{30} \mathrm{~S}_{10}$ $\mathrm{kg} \mathrm{ha}^{-1}$ can support for higher yields of fieldpea and help to sustain fertility of calcareous soils.
\end{abstract}

Keywords: Boron, calcareous soil, fieldpea productivity, nodulation, nutrient uptake, quality, Zinc.

\section{INTRODUCTION}

Pulses are rich source of protein and play a significant role in correcting the prevalent malnutrition in the country like Bangladesh and India (Singh et al., 2015). Among the pulses, fieldpea (Pisum sativum L.) is an important grain legume in the Asia, Europe, North America, Japan and Australia but in Bangladesh, fieldpea is going to be a major pulse crop within a few years (BBS, 2016). It is highly nutritive which contains high proportion of digestive protein
(20 to 22.5\%) (Singh et al., 2015). Fieldpea is cultivated in Bangladesh for fresh green seed, boiled green pods and dried seed. The green pods of pea may contribute to a major income sources for small-scale farmers (Musinguzi et al., 2010).

Besides, fieldpea improves the soil health through biological nitrogen fixation (about 30 to $50 \mathrm{~kg} \mathrm{~N} \mathrm{ha}^{-1}$ ) and addition of organic matter to the soil (Erman et al., 2009). 
The yield of pulses in Bangladesh is low due to many reasons: nutrient deficiency (including micronutrient) is one of them (Quddus et al., 2014). Nutrient deficiency in soil leads to reduction of pulses productivity (Kumar and Singh, 2009). The soils of different parts of Bangladesh particularly calcareous soils are more or less deficient in $\mathrm{Zn}$ and $\mathrm{B}$ as well as the population of nitrogen fixing bacteria (Rhizobium sp.) is less which are main cause of poor pulse yields (Jahiruddin, 2015; Quddus et al., 2014). General fertility level of calcareous soil is low to medium and the $\mathrm{Zn}$ and $\mathrm{B}$ status is medium or low (Rashid, 2001; FRG, 2012).

Zinc plays a vital role in metabolism and it is involved in $\mathrm{N}$-fixation through nodule formation (Patel et al., 2011). Zinc is required for pollen function and fertilization (Ali et al., 2017). Legume crops required more amount of boron for proper development of reproductive organs. Its deficiency leads to sterility in plants by malformation of reproductive tissues affecting pollen germination, resulting in increased flower drop and reduced fruit set (Subasinghe et al., 2003). Boron also plays a key role in sugar translocation, nitrogen fixation, protein synthesis, sucrose synthesis, cell wall composition, membrane stability, and $\mathrm{K}^{+}$transport (Singh et al., 2014).

Thus, the conjunctive use of Zinc and Boron including macro nutrients may create a great possibility to increase fieldpea production and soil fertility improvement. The positive impacts of these two micronutrients ( $Z n$ and $B)$ on several crops like chickpea, lentil, mungbean etc. have already been stated in Bangladesh and Indian subcontinent (Karan et al., 2014; Quddus et al., 2011). But very limited study has been done to evaluate the impact of $\mathrm{Zn}$ and $\mathrm{B}$ on fieldpea production. Therefore, the present study was undertaken to evaluate the effect of $\mathrm{Zn}$ and $\mathrm{B}$ on productivity, nutrient uptake and quality of fieldpea and soil fertility; and find out the suitable combination of $\mathrm{Zn}$ and $\mathrm{B}$ fertilization for maximium yield of fieldpea.

\section{MATERIALS AND METHODS}

\section{Site description and soils}

Field experiments were conducted for two consecutive years (winter season of 2014-15 and 2015-16) at two locations viz. (i) the research field of Regional Pulses Research Station (RPRS), Bangladesh Agricultural Research Institute (BARI), located in the moist monsoon

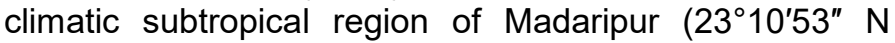
latitude and $90^{\circ} 11^{\prime} 28^{\prime \prime} \mathrm{E}$ longitude) at an elevation of 7.0 $\mathrm{m}$ above the sea level. The calcareous soils of Madaripur is medium high land with loamy texture which belongs to Gopalpur series (Soil taxonomy: Order-Inceptisols, SubGroup-Aquic Eutrochrepts) under the Low Ganges River Flood plain (Agro-Ecological Zone-12). Madaripur receives 1.0 to $83.0 \mathrm{~mm}$ rainfall which occurs from October to March. The mean minimum and maximum air temperatures during the period (October to March) of the experiment were 11.0 and $31.5^{\circ} \mathrm{C}$, respectively. Average air temperature varied from 20.2 to $29.1^{\circ} \mathrm{C}$ during $2014-15$ and 18.2 to $27.5^{\circ} \mathrm{C}$ during $2015-16$ (Figure 1). (ii) The Regional Agricultural Research Station (RARS) farm. RARS located in the Jashore $\left(23.11^{\circ} \mathrm{N}\right.$ latitude and $89.14^{\circ} \mathrm{E}$ longitude) lies at an elevation of $6.71 \mathrm{~m}$ above the sea level. The land belongs to High Ganges River Flood plain (Agro-Ecological Zone-11) and Gopalpur soil series (Soil taxonomy: Order-Inceptisols, Sub-Group-Aquic Eutrochrepts). The soils of Jashore are calcareous in nature having silt loam texture. The Jashore area receives average rainfall from 1.7 to $65.0 \mathrm{~mm}$ during October to March. The mean minimum and maximum air temperatures during the period (October to March) of the experiment were 9.30 and $30.6^{\circ} \mathrm{C}$, respectively. Average air temperatures ranged from 16.6 to $24.5^{\circ} \mathrm{C}$ during 2014 15 and 17.1 to $25.3^{\circ} \mathrm{C}$ during $2015-16$ (Figure 1 ). The initial soil samples $(0-15 \mathrm{~cm}$ depth) of both locations have been analysed and fertility status are presented in the Table 1.

\section{Land preparation, experimental treatment, design and fertilizer application}

The land was first opened by a tractor operated chisel plough and then prepared thoroughly by ploughing with a power tiller followed by laddering and leveling. The clods were broken and the fields were made weed and stubbles free. Hence, the experiment was planned with sixteen (16) treatment combinations comprising four levels of $\mathrm{Zn}(0,1.0$, 2.0 and $\left.3.0 \mathrm{~kg} \mathrm{ha}^{-1}\right)$ and four levels of $B(0,1.0,1.5$ and $2.0 \mathrm{~kg} \mathrm{ha}^{-1}$ ) along with a blanket dose of other fertilizers of $\mathrm{N}, \mathrm{P}, \mathrm{K}$ and $\mathrm{S}$ at $12,22,30$ and $10 \mathrm{~kg} \mathrm{ha}^{-1}$, respectively. The treatment combinations were as $T_{1}=Z_{n_{0}} B_{0} ; T_{2}=$ $Z n_{0} B_{1.0} ; T_{3}=Z n_{0} B_{1.5} ; T_{4}=Z n_{0} B_{2.0} ; T_{5}=Z n_{1.0} B_{0} ; T_{6}=$ $Z n_{1.0} B_{1.0} ; T_{7}=Z n_{1.0} B_{1.5} ; T_{8}=Z n_{1.0} B_{2.0} ; T_{9}=Z_{2.0} B_{0} ; T_{10}=$ $Z_{2.0} B_{1.0} ; T_{11}=Z n_{2.0} B_{1.5} ; T_{12}=Z n_{2.0} B_{2.0} ; T_{13}=Z_{3.0} B_{0} ; T_{14}=$ $Z n_{3.0} B_{1.0} ; \quad T_{15}=Z n_{3.0} B_{1.5}$; and $T_{16}=Z n_{3.0} B_{2.0}$. The experimental design was a split-plot with three replications. The unit plot size was $12 \mathrm{~m}^{2}(4 \mathrm{~m} \times 3 \mathrm{~m})$. Zinc and $\mathrm{B}$ were applied treatment wise as Zinc sulphate $\left(\mathrm{ZnSO}_{4} .7 \mathrm{H}_{2} \mathrm{O}\right)$ and Boric acid $\left(\mathrm{H}_{3} \mathrm{BO}_{3}\right)$, respectively. Every plot received an equal amount of other fertilizers at $\mathrm{N}_{12} \mathrm{P}_{22}$ $\mathrm{K}_{30} \mathrm{~S}_{10} \mathrm{~kg} \mathrm{ha}^{-1}$ (FRG, 2012) as urea, TSP, MoP and gypsum during final plot preparation. The unit plots were separated from each other by an alley of $50 \mathrm{~cm}$ width. Three replicated blocks were alienated by the space of 1 $\mathrm{m}$ width.

\section{Seed sowing and agronomic practices}

Seeds of a high yielding fieldpea variety (BARI Fieldpea1) were treated using the fungicide Provex 200 (at $2.5 \mathrm{~g}$ $\mathrm{kg}^{-1}$ seeds) before sowing for controlling of soil born diseases. Treated seeds were sown at $30 \mathrm{~kg} \mathrm{ha}^{-1}$ on 09 November, 2014 and 07 November, 2015 at Madaripur and 12 November, 2014 and 09 November, 2015 at 


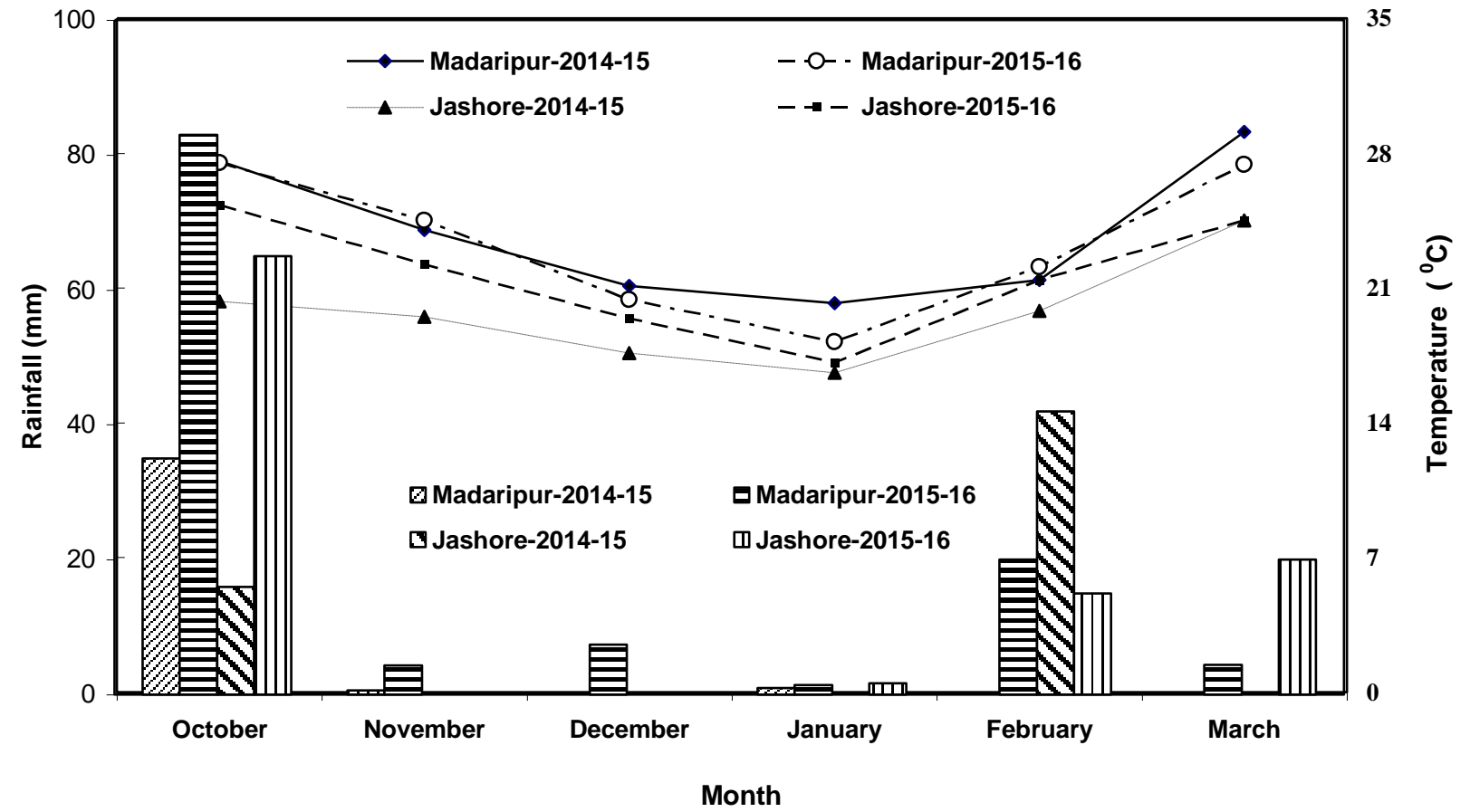

Figure 1. Rainfall and air temperatures during the experimental period at Madaripur and Jashore.

Table 1. Fertility status of initial soil sample of the experimental field at Madaripur and Jashore.

\begin{tabular}{|c|c|c|c|c|c|c|c|c|c|}
\hline \multirow{2}{*}{ Location } & \multirow{2}{*}{$\mathrm{pH}$} & \multirow{2}{*}{ OM (\%) } & \multirow{2}{*}{$\begin{array}{l}\text { Total } \\
\text { N (\%) }\end{array}$} & $\mathrm{Ca}$ & $\mathrm{K}$ & $\mathbf{P}$ & $S$ & $\mathrm{Zn}$ & B \\
\hline & & & & \multicolumn{2}{|c|}{$\begin{array}{ll}\text { Ca } & \mathrm{K} \\
\text { meq. } 100 \mathrm{~g}^{-1} \\
\end{array}$} & \multicolumn{4}{|c|}{$\mu g^{-1}$} \\
\hline Madaripur & 7.4 & 1.42 & 0.063 & 12.8 & 0.15 & 14.0 & 16.0 & 1. 18 & 0.15 \\
\hline Jashore & 8.3 & 1.64 & 0.072 & 16.7 & 0.16 & 14.6 & 16.5 & 1.10 & 0.16 \\
\hline Critical level & - & - & 0.12 & 2.0 & 0.12 & 10 & 10 & 0.60 & 0.20 \\
\hline Interpretation & $\begin{array}{l}\text { Slightly } \\
\text { alkaline }\end{array}$ & Low & $\begin{array}{l}\text { Very } \\
\text { low }\end{array}$ & $\begin{array}{l}\text { Very } \\
\text { high }\end{array}$ & Low & Low & Medium & Medium & Low \\
\hline
\end{tabular}

Jashore. Seeds were sown continuously in rows (10 rows/plot) maintaining row to row spacing of $30 \mathrm{~cm}$. Hand weeding as well as thining of seedlings were done at 25 days after sowing (DAS) maintaining the distance of plant to plant $05 \mathrm{~cm}$ by making a total of 800 plants per plot $(12$ $\mathrm{m}^{2}$ ). Again, hand weeding was done at 50 DAS. The disease (powdery mildew) was control by spraying of Thiovit fungicide @ $2 \mathrm{~g} \mathrm{~L}^{-1}$ in two times at 10 days interval, starting from flowering stage and insects (pod borer and aphid) were controlled by spraying insecticide of Karate @ $0.2 \%$ during two times at podding stage interval of 10 days. Mature crop means fieldpea plants to be prostrate vines and the seed become hard containing $14-15 \%$ moisture (Oelke et al., 1991).

\section{Data collection}

Number of nodules per plant was calculated at seedling, vegetative, flowering and podding stage at an interval of 15 days. During calculation 5 plants from each plot were selected randomly. Plants were smoothly uprooted and the soil from roots was removed carefully using tap water. The roots were then washed with distilled water, blotted with tissue paper and the number of nodules per plant was counted. Separated nodules were sliced into two pieces to observe the inside colour for nodules activity. The lightpink or red coloured nodules were considered as active.

Regarding pods per plant, 10 mature plants of fieldpea were randomly selected and uprooted from the middle eight rows of each plot at the harvest time. Pods were detached from every plant and the number of pods per plant was counted and average calculated. Then seeds were separated from selected 10 plants in each plot. For stover yield ( $\left.\mathrm{kg} \mathrm{ha}^{-1}\right)$, mature plants were collected from two $1 \mathrm{~m}^{2}$ quadrates in each plot at harvest time. The harvested plants were brought to the threshing floor for sun dry and seeds were separated from stover with the help of 
bamboo stick. The sun dried stovers were weighed and recorded data were converted to $\mathrm{kg} \mathrm{ha}^{-1}$. Rest of the matured plants (including border rows) of each plot were also harvested and brought to the threshing floor for sun drying and seeds were separated. The seed yield ( $\mathrm{kg} \mathrm{ha}$ 1) was calculated based on whole plot $(4 \mathrm{~m} \times 3 \mathrm{~m})$ technique. Thousand seed weight $(\mathrm{g})$ was determined by the counting of 500 seeds randomly from each plot and weighing through electronic balance and converting it into 1000 -seed weight adjusting at around $10 \%$ moisture content (Seedburo 1200D Digital Moisture Tester Manual, USA).

\section{Soil and plant analysis}

Treatment-wise soil samples (depth $0-15 \mathrm{~cm}$ ) were collected after crop harvest. The composite soil sample of each plot was brought to the laboratory and spread on a brown paper for air drying. The air-dried soil samples were grounded and passed through a 2-mm sieve. After sieving, the prepared soil samples were kept in plastic containers with proper label for chemical analysis. Soil $\mathrm{pH}$ was measured by glass electrode $\mathrm{pH}$ meter using soil: water ratio of 1:2.5 (Page et al., 1982) and organic matter by Nelson and Sommers (1982) method; total N by Microkjeldahl method (Bremner and Mulvaney, 1982); exchangeable $\mathrm{K}$ by $1 \mathrm{~N} \mathrm{NH}_{4} \mathrm{OAc}$ method (Jackson, 1973); exchangeable $\mathrm{Ca}$ by $1 \mathrm{~N} \mathrm{NH}_{4} \mathrm{OAc}$ method (Gupta, 2004); available $\mathrm{P}$ by Olsen and Sommers (1982) method; available $\mathrm{S}$ by turbidity method using $\mathrm{BaCl}_{2}$ (Fox et al., 1964); available Zn by DTPA method (Lindsay and Norvell, 1978); available B by azomethine-H method (Page et al., 1982).

Plant samples (stover and seed) from each plot were oven-dried at $70^{\circ} \mathrm{C}$ for $48 \mathrm{~h}$ and finely grounded by a Cyclotec $^{\mathrm{TM}} 1093$ sample Mill (Made in Sweden). Treatment wise grounded samples (100 g of stover and $100 \mathrm{~g}$ of seeds) were stored in polybag (size, $15 \mathrm{~cm} \times 10$ $\mathrm{cm}$ ) for analysis. Afterwards, $0.1 \mathrm{~g}$ of each of the grounded samples (stover and seeds) was analysed for $\mathrm{N}$ using the Kjeldahl method FOSS (Persson et al., 2008). Oven-dried each grounded samples of $0.5 \mathrm{~g}$ were taken into a $50 \mathrm{ml}$ digestion flask; $5 \mathrm{ml}$ of diacid mixture $\left(\mathrm{HNO}_{3}\right.$ and $\left.\mathrm{HClO}_{4}\right)$ as described by Piper (1966) was added to the flask. The flask was placed on hot plate and the temperature was raised upto $190^{\circ} \mathrm{C}$ and the digestion was done for 2 hours. The flask was then removed and allowed to cool upto room temperature. The samples were diluted with distilled water and filtered through a filter paper (Whatman No. 42) in a $100 \mathrm{ml}$ volumetric flask and volume was made up to the mark by adding distilled water. Zinc concentration in the digest was directly measured by Atomic Absorption Spectrophotometer (VARIAN SpectrAA 55B, Australia). Boron concentration was determined by spectrophotometer following azomethine- $\mathrm{H}$ method (Page et al., 1982).
Protein contein in fieldpea seed was estimated by multiplying the $\% \mathrm{~N}$ content of seed with pulses food factor 5.30 (FAO, 2018). Crop uptake of $\mathrm{Zn}$ and B was estimated by multiplying the total dry matter yields of fieldpea with corresponding Zn and B concentrations (Quddus et al., 2017).

\section{Statistical analysis}

Data of nodules per plant, N, Zn, B, protein content and nutrient uptake ( $\mathrm{Zn}$ and $\mathrm{B}$ ) were computed as an averaged of two study years. Averaged data and data of yields (seed and stover yield) and yield attributes (number of pods per plant and 1000 seed weight) were statistical analysed through ANOVA procedure using a split-plot design with three replicates considering main-plot factor $\mathrm{Zn}$ and subplot factor B. Then multiple comparisons like all-pairwise comparisons i.e. the means of treatment tested by LSD method at 5\% (LSD0.05) level of significance (Statistix 10., 1985).

\section{RESULTS}

\section{Effect of Zinc and Boron on yields and yield attributes}

Results from Table 2 reveal that different combinations of $\mathrm{Zn}$ and $\mathrm{B}$ contributed significantly to get higher yields of fieldpea in both years in Madaripur and Jashore. The mean seed yield (average of two years) varied among the different combinations of $\mathrm{Zn}$ and $\mathrm{B}$ from $889 \mathrm{~kg} \mathrm{ha}^{-1}$ to $1567 \mathrm{~kg} \mathrm{ha}^{-1}$ at Madaripur and from $983 \mathrm{~kg} \mathrm{ha}^{-1}$ to $1615 \mathrm{~kg}$ $\mathrm{ha}^{-1}$ at Jashore (Figure 2). The highest seed yield $(1581 \mathrm{~kg}$ $\mathrm{ha}^{-1}$ in $1 \mathrm{st}$ year and $1553 \mathrm{~kg} \mathrm{ha}^{-1}$ in 2nd year) at Madaripur was found in the treatment $Z n_{3.0} B_{2.0}$ which was statistically similar to treatment $Z_{2.0} B_{2.0}$ and $Z n_{3.0} B_{1.5}$ in 1 st year and only $\mathrm{Zn}_{3.0} \mathrm{~B}_{1.5}$ in 2 nd year. Significantly, highest seed yield of $1631 \mathrm{~kg} \mathrm{ha}^{-1}$ in 1 st year and $1598 \mathrm{~kg} \mathrm{ha}^{-1}$ in 2nd year at Jashore was found in the treatment $\mathrm{Zn}_{3.0} \mathrm{~B}_{2.0}$ followed by the treatments $Z n_{3.0} B_{1.5}$ in $2 n d$ year and $Z n_{3.0} B_{1.0}$ and $Z_{3.0} B_{1.5}$ in 1 st year. Among the treatments, the lowest seed yield was observed in control treatment of $Z n_{0} B_{0}$ in both years and locations (Table 2). The stover yield (mean of two years) of fieldpea ranged from 2457 to $3721 \mathrm{~kg}$ ha${ }^{1}$ at Madaripur and 2536 to $3955 \mathrm{~kg} \mathrm{ha}^{-1}$ at Jashore (Figure 2). The treatment combination of $\mathrm{Zn}_{3.0} \mathrm{~B}_{2.0}$ showed significant highest stover yield of fieldpea in both years and locations though there were few exceptions (Table 2). The seed yield was found comperatively higher in Jashore than in Madaripur (Figure 2). This variation might be due to variation in soil fertility and weather differences between the locations.

Yield attributes of fieldpea viz. number pods per plant and 1000 seed weight were influenced significantly by the combine application of $\mathrm{Zn}$ and $\mathrm{B}$ at both locations (Table 3). Maximum number of pods per plant was obtained in 
Table 2. Effect of zinc and boron on seed yield and stover yield of fieldpea.

\begin{tabular}{|c|c|c|c|c|c|c|c|c|}
\hline \multirow{3}{*}{ Treatments } & \multicolumn{4}{|c|}{ Seed yield (kg ha-1) } & \multicolumn{4}{|c|}{ Stover yield $\left(\mathrm{kg} \mathrm{ha}^{-1}\right)$} \\
\hline & \multicolumn{2}{|c|}{ Madaripur } & \multicolumn{2}{|c|}{ Jashore } & \multicolumn{2}{|c|}{ Madaripur } & \multicolumn{2}{|c|}{ Jashore } \\
\hline & 1st yr & 2nd yr & 1st yr & 2nd yr & 1st yr & 2nd yr & $1 \mathrm{st} \mathrm{yr}$ & 2nd yr \\
\hline $\mathrm{Zn}_{0} \mathrm{~B}_{0}$ & $909^{f}$ & $869^{i}$ & $1015^{g}$ & $950^{j}$ & $2585^{e}$ & $2329^{h}$ & $2578^{k}$ & $2494^{i}$ \\
\hline $\mathrm{Zn}_{0} \mathrm{~B}_{1.0}$ & $1164^{\mathrm{c}-\mathrm{e}}$ & $1047^{\mathrm{h}}$ & $1306^{\mathrm{ef}}$ & $1098^{i}$ & $3026^{c-e}$ & $2727^{g}$ & $2912^{i}$ & $2896^{h}$ \\
\hline $\mathrm{Zn}_{0} \mathrm{~B}_{1.5}$ & $1156^{\text {de }}$ & $1104^{\mathrm{h}}$ & $1400^{c-e}$ & $1146^{\mathrm{hi}}$ & $2973^{\text {de }}$ & $2858^{g}$ & $2965^{\text {hi }}$ & $2917^{\text {gh }}$ \\
\hline $\mathrm{Zn}_{0} \mathrm{~B}_{2.0}$ & $1182^{c-e}$ & $1178^{g}$ & $1492^{\mathrm{a}-\mathrm{e}}$ & $1225^{g h}$ & $3022^{c-e}$ & $3093^{f}$ & $3054^{g h}$ & $3061^{\mathrm{f}-\mathrm{h}}$ \\
\hline $\mathrm{Zn}_{1.0} \mathrm{~B}_{0}$ & $1058^{\text {ef }}$ & $1093^{h}$ & $1128^{f g}$ & $1221^{\mathrm{gh}}$ & $2767^{\text {de }}$ & 28399 & $2768^{j}$ & $3030^{f-h}$ \\
\hline $\mathrm{Zn}_{1.0} \mathrm{~B}_{1.0}$ & $1174^{\mathrm{c}-\mathrm{e}}$ & $1240^{\mathrm{e}-\mathrm{g}}$ & $1422^{\mathrm{b}-\mathrm{e}}$ & $1309^{f g}$ & $2842^{\text {de }}$ & $3262^{\text {def }}$ & $3078^{g}$ & $3321^{\text {ef }}$ \\
\hline $\mathrm{Zn}_{1.0} \mathrm{~B}_{1.5}$ & $1216^{\mathrm{c}-\mathrm{e}}$ & $1276^{d-f}$ & $1453^{a-e}$ & $1371^{\text {ef }}$ & $3065^{c-e}$ & $3394^{\mathrm{cd}}$ & $3356^{f}$ & $3593^{\text {de }}$ \\
\hline $\mathrm{Zn}_{1.0} \mathrm{~B}_{2.0}$ & $1211^{\mathrm{c}-\mathrm{e}}$ & $1319^{\mathrm{cd}}$ & $1569^{a-c}$ & $1453^{c-e}$ & $3014^{c-e}$ & $3448^{\mathrm{bcd}}$ & $3410^{\text {ef }}$ & $3690^{c d}$ \\
\hline $\mathrm{Zn}_{2.0} \mathrm{~B}_{0}$ & $1173^{\mathrm{c}-\mathrm{e}}$ & $1210^{f g}$ & $1367^{\text {de }}$ & $1300^{f g}$ & $2994^{\text {c-e }}$ & $3160^{\text {ef }}$ & $3387^{\text {ef }}$ & $3244^{e-h}$ \\
\hline $\mathrm{Zn}_{2.0} \mathrm{~B}_{1.0}$ & $1324^{\mathrm{bc}}$ & $1308^{c-e}$ & $1536^{a-d}$ & $1427^{\text {de }}$ & $3288^{a-d}$ & $3363^{\text {cde }}$ & $3464^{\text {de }}$ & $3683^{c d}$ \\
\hline $\mathrm{Zn}_{2.0} \mathrm{~B}_{1.5}$ & $1288^{b-d}$ & $1364^{c}$ & $1481^{\mathrm{a}-\mathrm{e}}$ & $1478^{\mathrm{b}-\mathrm{e}}$ & $3102^{\mathrm{b}-\mathrm{e}}$ & $3517^{a b c}$ & $3324^{f}$ & $3830^{\mathrm{b}-\mathrm{d}}$ \\
\hline $\mathrm{Zn}_{2.0} \mathrm{~B}_{2.0}$ & $1465^{\mathrm{ab}}$ & $1434^{b}$ & $1528^{a-d}$ & $1541^{a-c}$ & $3615^{a b}$ & $3543^{a b c}$ & $3521^{\mathrm{bcd}}$ & $3988^{a-c}$ \\
\hline $\mathrm{Zn}_{3.0} \mathrm{~B}_{0}$ & $1182^{\mathrm{c}-\mathrm{e}}$ & $1244^{\mathrm{e}-\mathrm{g}}$ & $1414^{\mathrm{b}-\mathrm{e}}$ & $1284^{\mathrm{fg}}$ & $3017^{\text {c-e }}$ & $3270^{\text {def }}$ & $3479^{\text {cde }}$ & $3279^{--g}$ \\
\hline $\mathrm{Zn}_{3.0} \mathrm{~B}_{1.0}$ & $1188^{\mathrm{c}-\mathrm{e}}$ & $1441^{\mathrm{b}}$ & $1603^{a b}$ & $1489^{b-d}$ & $3029^{c-e}$ & $3532^{\mathrm{abc}}$ & $3577^{a b c}$ & $3856^{\text {b-d }}$ \\
\hline $\mathrm{Zn}_{3.0} \mathrm{~B}_{1.5}$ & $1413^{a b}$ & $1526^{a}$ & $1567^{a-c}$ & $1578^{a b}$ & $3533^{a-c}$ & $3626^{a b}$ & $3605^{a b}$ & $4095^{a b}$ \\
\hline $\mathrm{Zn}_{3.0} \mathrm{~B}_{2.0}$ & $1581^{a}$ & $1553^{a}$ & $1631^{a}$ & $1598^{a}$ & $3715^{a}$ & $3726^{a}$ & $3647^{a}$ & $4262^{a}$ \\
\hline CV (\%) & 8.64 & 2.94 & 7.76 & 4.80 & 11.1 & 4.00 & 2.02 & 5.97 \\
\hline LSD $(0.05)$ & 179 & 62.7 & 185 & 109 & 542 & 218 & 111 & 348 \\
\hline
\end{tabular}

Values within the same column with a common letter do not differ significantly $(P<0.05)$.
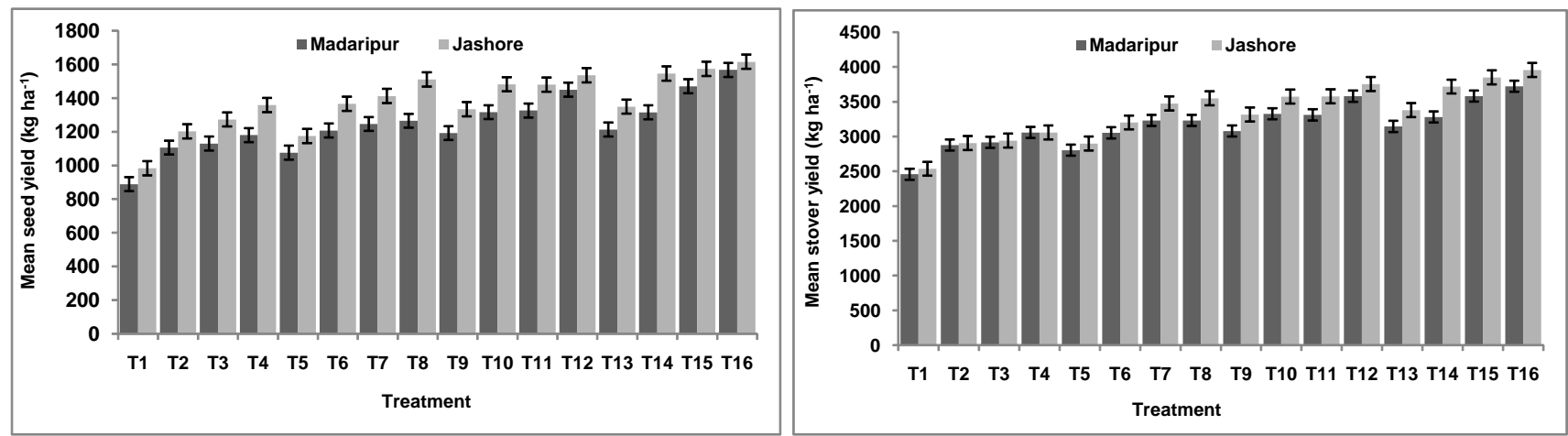

Figure 2. Effectof $Z n$ and $B$ on mean seed yield and mean stover yield of fieldpea. Note: Errors bars represent the SEM. Treatment: $\mathrm{T}_{1}=\mathrm{Zn}_{0} \mathrm{~B}_{0} ; \mathrm{T}_{2}=\mathrm{Zn}_{0} \mathrm{~B}_{1.0} ; \mathrm{T}_{3}=\mathrm{Zn}_{0} \mathrm{~B}_{1.5} ; \mathrm{T}_{4}=\mathrm{Zn}_{0} \mathrm{~B}_{2.0} ; \mathrm{T}_{5}=\mathrm{Zn}_{1.0} \mathrm{~B}_{0} ; \mathrm{T}_{6}=\mathrm{Zn}_{1.0} \mathrm{~B}_{1.0} ; \mathrm{T}_{7}=\quad \mathrm{Zn}_{1.0} \mathrm{~B}_{1.5} ; \mathrm{T}_{8}=\mathrm{Zn}_{1.0} \mathrm{~B}_{2.0} ; \mathrm{T}_{9}=$ $\mathrm{Zn}_{2.0} \mathrm{~B}_{0} ; \mathrm{T}_{10}=\mathrm{Zn}_{2.0} \mathrm{~B}_{1.0} ; \mathrm{T}_{11}=\mathrm{Zn}_{2.0} \mathrm{~B}_{1.5} ; \mathrm{T}_{12}=\mathrm{Zn}_{2.0} \mathrm{~B}_{2.0} ; \mathrm{T}_{13}=\mathrm{Zn}_{3.0} \mathrm{~B}_{0} ; \mathrm{T}_{14}=\mathrm{Zn}_{3.0} \mathrm{~B}_{1.0} ; \mathrm{T}_{15}=\mathrm{Zn}_{3.0} \mathrm{~B}_{1.5} ;$ and $\mathrm{T}_{16}=\mathrm{Zn}_{3.0} \mathrm{~B}_{2.0} \mathrm{~kg}$ ha ${ }^{-1}$, respectively.

treatment $\mathrm{Zn}_{3.0} \mathrm{~B}_{2.0}$ followed by $\mathrm{Zn}_{3.0} \mathrm{~B}_{1.5}$ in both the years at Madaripur, but in1st year at Jashore, the maximum pods per plant was obtained in treatment $Z n_{3.0} B_{1.5}$ followed by $\mathrm{Zn}_{3.0} \mathrm{~B}_{2.0}$ and $\mathrm{Zn}_{2.0} \mathrm{~B}_{2.0}$. The highest 1000 seed weight was observed in treatment $\mathrm{Zn}_{3.0} \mathrm{~B}_{2.0}$ which was significantly different among the treatments at Madaripur and Jashore, but in 1st year at Jashore significantly highest 1000 seed weight of fieldpea was recorded in treatment $\mathrm{Zn}_{3.0} \mathrm{~B}_{1.5}$. However, the lowest pods per plant and 1000 seed weight were recorded in treatment $\mathrm{Zn}_{0} \mathrm{~B}_{0}$. Combine application of
$\mathrm{Zn}$ and $\mathrm{B}$ showed an increasing trend on number pods per plant and 1000 seed weight of fieldpea than their single application (Table 3).

\section{Effect of Zinc and Boron on nodulation}

Combine application of $\mathrm{Zn}$ and $\mathrm{B}$ contributed significantly to produce higher number of nodules per plant of fieldpea during different dates of sampling (Table 4). Number of 
Table 3. Effect of zinc and boron on yield attributes of fieldpea.

\begin{tabular}{|c|c|c|c|c|c|c|c|c|}
\hline \multirow{3}{*}{ Treatments } & \multicolumn{4}{|c|}{ No. of Pods plant ${ }^{-1}$} & \multicolumn{4}{|c|}{1000 seeds wt $(g)$} \\
\hline & \multicolumn{2}{|c|}{ Madaripur } & \multicolumn{2}{|c|}{ Jashore } & \multicolumn{2}{|c|}{ Madaripur } & \multicolumn{2}{|c|}{ Jashore } \\
\hline & 1st yr & 2nd yr & 1st yr & 2nd yr & 1st yr & 2nd yr & 1st yr & 2nd yr \\
\hline $\mathrm{Zn}_{0} \mathrm{~B}_{0}$ & $16.1^{\mathrm{g}}$ & $12.0^{\mathrm{j}}$ & $23.3^{d}$ & 17.9 & $63.9^{b}$ & $61.2^{\mathrm{g}}$ & $59.9^{f}$ & $48.6^{\mathrm{i}}$ \\
\hline $\mathrm{Zn}_{0} \mathrm{~B}_{1.0}$ & $21.1^{\text {def }}$ & $15.1^{i}$ & $26.3^{\mathrm{cd}}$ & 21.8 & $64.8^{\mathrm{ab}}$ & $62.2^{\text {ef }}$ & $52.1^{\mathrm{d}-\mathrm{f}}$ & $50.3^{h}$ \\
\hline $\mathrm{Zn}_{0} \mathrm{~B}_{1.5}$ & $19.7^{\mathrm{d}-\mathrm{g}}$ & $16.6^{\mathrm{hi}}$ & $28.7^{\mathrm{ac}}$ & 22.3 & $64.1^{\mathrm{ab}}$ & $62.4^{\text {def }}$ & $53.2^{c-f}$ & $50.9^{g h}$ \\
\hline $\mathrm{Zn}_{0} \mathrm{~B}_{2.0}$ & $20.7^{\text {def }}$ & $17.8^{g h}$ & $32.7^{\mathrm{ab}}$ & 23.9 & $64.7^{\mathrm{ab}}$ & $62.6^{\mathrm{b}-\mathrm{e}}$ & $53.2^{c-f}$ & $51.6^{e-g}$ \\
\hline $\mathrm{Zn}_{1.0} \mathrm{~B}_{0}$ & $18.1^{\mathrm{fg}}$ & $14.7^{i}$ & $30.3^{a-c}$ & 22.5 & $64.3^{\mathrm{ab}}$ & $62.1^{f}$ & $51.2^{\text {ef }}$ & $51.2^{\mathrm{f}-\mathrm{h}}$ \\
\hline $\mathrm{Zn}_{1.0} \mathrm{~B}_{1.0}$ & $20.3^{\text {def }}$ & $19.4^{e-g}$ & $30.3^{a-c}$ & 24.3 & $64.6^{\mathrm{ab}}$ & $62.6^{b-f}$ & $55.7^{a-c}$ & $52.2^{\mathrm{c}-\mathrm{e}}$ \\
\hline $\mathrm{Zn}_{1.0} \mathrm{~B}_{1.5}$ & $22.5^{\text {de }}$ & $21.1^{\mathrm{c}-\mathrm{e}}$ & $31.3^{\mathrm{a}-\mathrm{c}}$ & 25.7 & $64.2^{\mathrm{ab}}$ & $62.9^{a b c}$ & $54.0^{\mathrm{a}-\mathrm{e}}$ & $52.6^{\mathrm{b}-\mathrm{d}}$ \\
\hline $\mathrm{Zn}_{1.0} \mathrm{~B}_{2.0}$ & $19.1^{\text {efg }}$ & $21.5^{\mathrm{cd}}$ & $32.7^{\mathrm{ab}}$ & 27.8 & $65.1^{\mathrm{a}}$ & $63.0^{\mathrm{ab}}$ & $54.8^{\mathrm{a}-\mathrm{d}}$ & $52.8^{\mathrm{b}-\mathrm{d}}$ \\
\hline $\mathrm{Zn}_{2.0} \mathrm{~B}_{0}$ & $19.0^{\text {efg }}$ & $16.5^{\mathrm{hi}}$ & $29.0^{a-c}$ & 24.9 & $64.4^{\mathrm{ab}}$ & $62.1^{\dagger}$ & $53.1^{\mathrm{c}-\mathrm{f}}$ & $52.2^{\mathrm{c}-\mathrm{e}}$ \\
\hline $\mathrm{Zn}_{2.0} \mathrm{~B}_{1.0}$ & $26.8^{\mathrm{bc}}$ & $19.1^{e-g}$ & $32.3^{a b}$ & 26.5 & $64.3^{\mathrm{ab}}$ & $62.7^{\mathrm{a}-\mathrm{e}}$ & $53.8^{\mathrm{a}-\mathrm{e}}$ & $52.9^{a-c}$ \\
\hline $\mathrm{Zn}_{2.0} \mathrm{~B}_{1.5}$ & $23.2^{\mathrm{cd}}$ & $20.3^{d-f}$ & $28.3^{b-d}$ & 28.0 & $64.7^{\mathrm{ab}}$ & $63.0^{\mathrm{ab}}$ & $53.5^{\mathrm{b}-\mathrm{e}}$ & $53.1^{\mathrm{ab}}$ \\
\hline $\mathrm{Zn}_{2.0} \mathrm{~B}_{2.0}$ & $28.3^{b}$ & $22.2^{\mathrm{bc}}$ & $33.0^{\mathrm{ab}}$ & 28.9 & $64.3^{\mathrm{ab}}$ & $63.2^{\mathrm{a}}$ & $53.7^{\mathrm{b}-\mathrm{e}}$ & $53.4^{\mathrm{ab}}$ \\
\hline $\mathrm{Zn}_{3.0} \mathrm{~B}_{0}$ & $23.0^{\text {cde }}$ & $18.6^{\text {fgh }}$ & $29.3^{a-c}$ & 25.7 & $64.1^{\mathrm{ab}}$ & $62.5^{c-f}$ & $52.8^{\mathrm{c}-\mathrm{f}}$ & $51.9^{\mathrm{d}-\mathrm{f}}$ \\
\hline $\mathrm{Zn}_{3.0} \mathrm{~B}_{1.0}$ & $21.8^{\text {def }}$ & $22.2^{b-d}$ & $31.7^{\mathrm{ab}}$ & 28.7 & $64.2^{\mathrm{ab}}$ & $62.9^{a-d}$ & $56.8^{\mathrm{ab}}$ & $53.2^{\mathrm{ab}}$ \\
\hline $\mathrm{Zn}_{3.0} \mathrm{~B}_{1.5}$ & $28.5^{\mathrm{b}}$ & $23.9^{a b}$ & $34.0^{\mathrm{a}}$ & 29.5 & $64.7^{\mathrm{ab}}$ & $63.0^{\mathrm{ab}}$ & $57.3^{a}$ & $53.4^{\mathrm{ab}}$ \\
\hline $\mathrm{Zn}_{3.0} \mathrm{~B}_{2.0}$ & $35.4^{\mathrm{a}}$ & $25.1^{\mathrm{a}}$ & $33.0^{\mathrm{ab}}$ & 30.9 & $64.9^{a}$ & $63.2^{\mathrm{a}}$ & $56.1^{a-c}$ & $53.7^{\mathrm{a}}$ \\
\hline CV (\%) & 5.91 & 5.72 & 7.33 & 5.01 & 1.05 & 0.49 & 3.81 & 0.89 \\
\hline LSD (0.05) & 4.17 & 1.85 & 4.73 & ns & 1.15 & 0.52 & 3.64 & 0.78 \\
\hline
\end{tabular}

Values within the same column with a common letter do not differ significantly $(P<0.05)$.

Table 4. Effect of zinc and boron on number of nodule per plant of fieldpea in different dates (pooled data of two years).

\begin{tabular}{|c|c|c|c|c|c|c|c|c|}
\hline \multirow{2}{*}{ Treatments } & \multicolumn{2}{|c|}{ No. nodule after 32 days } & \multicolumn{2}{|c|}{ No. nodule after 47 days } & \multicolumn{2}{|c|}{ No. nodule after 62 days } & \multicolumn{2}{|c|}{ No. nodule after 77 days } \\
\hline & Madaripur & Jashore & Madaripur & Jashore & Madaripur & Jashore & Madaripur & Jashore \\
\hline $\mathrm{Zn}_{0} \mathrm{~B}_{0}$ & $8.57^{9}$ & $15.6^{g}$ & $23.6^{9}$ & $30.5^{\mathrm{jk}}$ & $30.6^{9}$ & $41.3^{e f}$ & $22.6^{b-e}$ & $29.8^{\text {cde }}$ \\
\hline $\mathrm{Zn}_{0} \mathrm{~B}_{1.0}$ & $9.00^{9}$ & $16.2^{\mathrm{fg}}$ & $24.2 f^{g}$ & $29.9^{k}$ & $31.2^{\mathrm{fg}}$ & $42.0^{\text {def }}$ & $21.7^{\text {cde }}$ & $28.7^{\text {cde }}$ \\
\hline $\mathrm{Zn}_{0} \mathrm{~B}_{1.5}$ & $10.1^{\dagger}$ & $16.7^{\text {efg }}$ & $24.8^{\text {efg }}$ & $32.6^{\mathrm{hi}}$ & $30.9^{9}$ & $43.2^{c-f}$ & $22.8^{\text {cde }}$ & $27.6^{\mathrm{ef}}$ \\
\hline $\mathrm{Zn}_{0} \mathrm{~B}_{2.0}$ & $11.1^{\dagger}$ & $16.8^{\mathrm{d}-\mathrm{g}}$ & $25.4^{\mathrm{d}-\mathrm{g}}$ & $32.4^{\mathrm{hi}}$ & $32.4^{\text {efg }}$ & $42.8^{c-f}$ & $23.6^{\mathrm{a}-\mathrm{d}}$ & $29.1^{\text {cde }}$ \\
\hline $\mathrm{Zn}_{1.0} \mathrm{~B}_{0}$ & $10.2^{\dagger}$ & $15.6^{g}$ & $23.4^{\mathrm{g}}$ & $33.5^{\mathrm{gh}}$ & $30.7^{9}$ & $41.0^{f}$ & $21.6^{\mathrm{cde}}$ & $28.7^{\text {cde }}$ \\
\hline $\mathrm{Zn}_{1.0} \mathrm{~B}_{1.0}$ & $12.3^{\mathrm{e}}$ & $17.1^{\mathrm{d}-\mathrm{g}}$ & $25.4^{\mathrm{d}-\mathrm{g}}$ & $34.5^{\mathrm{fg}}$ & $33.6^{\text {def }}$ & $42.9^{c-f}$ & $20.9^{\text {de }}$ & $29.5^{\text {cde }}$ \\
\hline $\mathrm{Zn}_{1.0} \mathrm{~B}_{1.5}$ & $12.8^{\mathrm{de}}$ & $18.3^{a-f}$ & $26.7^{\text {cde }}$ & $35.7^{\text {ef }}$ & $34.4^{\text {cde }}$ & $42.8^{c-f}$ & $23.5^{\mathrm{a}-\mathrm{d}}$ & $25.7^{f}$ \\
\hline $\mathrm{Zn}_{1.0} \mathrm{~B}_{2.0}$ & $13.4^{d}$ & $18.5^{\mathrm{a}-\mathrm{e}}$ & $26.3^{\text {def }}$ & $36.4^{\text {de }}$ & $34.8^{\text {cde }}$ & $43.6^{c-f}$ & $22.9^{b-e}$ & $28.7^{\text {cde }}$ \\
\hline $\mathrm{Zn}_{2.0} \mathrm{~B}_{0}$ & $12.1^{\mathrm{e}}$ & $17.4^{\mathrm{b}-\mathrm{g}}$ & $24.5^{\mathrm{efg}}$ & $32.6^{\mathrm{hi}}$ & $33.7^{\mathrm{de}}$ & $42.7^{c-f}$ & $23.9^{a-d}$ & $29.8^{\text {cde }}$ \\
\hline $\mathrm{Zn}_{2.0} \mathrm{~B}_{1.0}$ & $14.5^{\mathrm{c}}$ & $18.5^{\mathrm{a}-\mathrm{e}}$ & $26.5^{\text {cde }}$ & $35.4^{\text {ef }}$ & $35.1^{\mathrm{cd}}$ & $44.3^{\mathrm{b}-\mathrm{e}}$ & $22.8^{\mathrm{b}-\mathrm{e}}$ & $27.9^{\text {def }}$ \\
\hline $\mathrm{Zn}_{2.0} \mathrm{~B}_{1.5}$ & $15.6^{\mathrm{ab}}$ & $18.9^{a-d}$ & $27.4^{\mathrm{cd}}$ & $36.9^{\mathrm{de}}$ & $36.4^{\mathrm{bc}}$ & $44.9^{b c d}$ & $19.8^{e}$ & $28.6^{\text {cde }}$ \\
\hline $\mathrm{Zn}_{2.0} \mathrm{~B}_{2.0}$ & $15.9^{a b}$ & $19.7^{\mathrm{ab}}$ & $28.6^{\mathrm{bc}}$ & $37.6^{\mathrm{cd}}$ & $36.7^{\mathrm{bc}}$ & $45.7^{\mathrm{bc}}$ & $25.7^{\mathrm{ab}}$ & $30.7^{\mathrm{bc}}$ \\
\hline $\mathrm{Zn}_{3.0} \mathrm{~B}_{0}$ & $13.5^{\mathrm{d}}$ & $17.3^{\mathrm{c}-g}$ & $25.9^{\text {def }}$ & $31.4^{\mathrm{ij}}$ & $34.9^{\mathrm{cd}}$ & $40.9^{f}$ & $23.8^{\mathrm{a}-\mathrm{d}}$ & $32.6^{\mathrm{ab}}$ \\
\hline $\mathrm{Zn}_{3.0} \mathrm{~B}_{1.0}$ & $14.6^{c}$ & $18.5^{\mathrm{a}-\mathrm{e}}$ & $28.7^{b c}$ & $38.7^{\mathrm{bc}}$ & $35.8^{\mathrm{bcd}}$ & $41.8^{\text {def }}$ & $26.1^{a}$ & $29.9^{\mathrm{cd}}$ \\
\hline $\mathrm{Zn}_{3.0} \mathrm{~B}_{1.5}$ & $15.2^{\mathrm{bc}}$ & $19.3^{\mathrm{abc}}$ & $29.8^{b}$ & $39.7^{\mathrm{ab}}$ & $37.8^{\mathrm{b}}$ & $47.3^{\mathrm{ab}}$ & $22.4^{\text {cde }}$ & $33.7^{\mathrm{a}}$ \\
\hline $\mathrm{Zn}_{3.0} \mathrm{~B}_{2.0}$ & $16.3^{a}$ & $20.1^{\mathrm{a}}$ & $32.7^{\mathrm{a}}$ & $40.5^{a}$ & $46.8^{a}$ & $49.8^{a}$ & $24.5^{\mathrm{abc}}$ & $32.9^{a b}$ \\
\hline CV (\%) & 4.79 & 7.98 & 5.42 & 2.68 & 4.26 & 4.56 & 8.95 & 4.91 \\
\hline LSD $(0.05)$ & 1.04 & 2.39 & 2.42 & 1.58 & 2.50 & 3.34 & 3.47 & 2.29 \\
\hline
\end{tabular}

Values within the same column with a common letter do not differ significantly $(P<0.05)$. 
Table 5. Effect of zinc and boron on protein content in seed and Zn \& B uptake by fieldpea (grain+stover) (pooled data of two years).

\begin{tabular}{|c|c|c|c|c|c|c|}
\hline \multirow{2}{*}{ Treatments } & \multicolumn{2}{|c|}{$\%$ Protein content in seed } & \multicolumn{2}{|c|}{ Zn uptake (kg ha-1) } & \multicolumn{2}{|c|}{ B uptake (kg ha-1) } \\
\hline & Madaripur & Jashore & Madaripur & Jashore & Madaripur & Jashore \\
\hline $\mathrm{Zn}_{0} \mathrm{~B}_{0}$ & $18.8^{b}$ & $18.3^{d}$ & $0.081^{h}$ & $0.082^{i}$ & $0.097^{i}$ & $0.103^{\mathrm{i}}$ \\
\hline $\mathrm{Zn}_{0} \mathrm{~B}_{1.0}$ & $19.3^{b}$ & $18.4^{d}$ & $0.097^{\text {fgh }}$ & $0.096^{\mathrm{hi}}$ & $0.114^{\text {ghi }}$ & $0.122^{\mathrm{hi}}$ \\
\hline $\mathrm{Zn}_{0} \mathrm{~B}_{1.5}$ & $19.4^{b}$ & $18.8^{\mathrm{cd}}$ & $0.098^{\text {fgh }}$ & $0.099^{g h i}$ & $0.119^{\text {fgh }}$ & $0.125^{\mathrm{ghi}}$ \\
\hline $\mathrm{Zn}_{0} \mathrm{~B}_{2.0}$ & $19.0^{\mathrm{b}}$ & $19.2^{\mathrm{cd}}$ & $0.109^{d-g}$ & $0.107^{\text {fgh }}$ & $0.127^{\mathrm{d}-\mathrm{h}}$ & $0.134^{\text {fgh }}$ \\
\hline $\mathrm{Zn}_{1.0} \mathrm{~B}_{0}$ & $19.5^{\mathrm{b}}$ & $19.0^{\mathrm{cd}}$ & $0.092^{\mathrm{gh}}$ & $0.094^{\mathrm{hi}}$ & $0.109^{h i}$ & $0.118^{\mathrm{hi}}$ \\
\hline $\mathrm{Zn}_{1.0} \mathrm{~B}_{1.0}$ & $20.6^{\mathrm{ab}}$ & $19.9^{b c d}$ & $0.110^{\mathrm{d}-\mathrm{g}}$ & $0.111^{\mathrm{e}-\mathrm{h}}$ & $0.124^{\mathrm{e}-\mathrm{h}}$ & $0.139^{e-h}$ \\
\hline $\mathrm{Zn}_{1.0} \mathrm{~B}_{1.5}$ & $19.9^{b}$ & $20.4^{\mathrm{bcd}}$ & $0.119^{c-g}$ & $0.122^{c-f}$ & $0.134^{\text {def }}$ & $0.152^{c-f}$ \\
\hline $\mathrm{Zn}_{1.0} \mathrm{~B}_{2.0}$ & $20.9^{\mathrm{ab}}$ & $20.8^{a-d}$ & $0.120^{c-f}$ & $0.129^{c-f}$ & $0.136^{\text {def }}$ & $0.160^{\mathrm{b}-\mathrm{e}}$ \\
\hline $\mathrm{Zn}_{2.0} \mathrm{~B}_{0}$ & $20.4^{\mathrm{ab}}$ & $19.9^{\mathrm{bcd}}$ & $0.108^{\mathrm{e}-\mathrm{h}}$ & $0.112^{\mathrm{d}-\mathrm{h}}$ & $0.124^{\mathrm{e}-\mathrm{h}}$ & $0.140^{d-h}$ \\
\hline $\mathrm{Zn}_{2.0} \mathrm{~B}_{1.0}$ & $21.0^{\mathrm{ab}}$ & $21.0^{\mathrm{a}-\mathrm{d}}$ & $0.126^{b-e}$ & $0.130^{\text {cde }}$ & $0.137^{c-f}$ & $0.160^{\mathrm{b}-\mathrm{e}}$ \\
\hline $\mathrm{Zn}_{2.0} \mathrm{~B}_{1.5}$ & $21.8^{a b}$ & $21.1^{a-d}$ & $0.128^{\mathrm{b}-\mathrm{e}}$ & $0.134^{\mathrm{bcd}}$ & $0.141^{\text {cde }}$ & $0.163^{b c d}$ \\
\hline $\mathrm{Zn}_{2.0} \mathrm{~B}_{2.0}$ & $22.3^{a b}$ & $21.7^{a b c}$ & $0.140^{a b c}$ & $0.143^{a b c}$ & $0.156^{b c}$ & $0.172^{a b c}$ \\
\hline $\mathrm{Zn}_{3.0} \mathrm{~B}_{0}$ & $20.9^{a b}$ & $20.5^{\mathrm{bcd}}$ & $0.116^{\mathrm{c}-\mathrm{g}}$ & $0.121^{c-g}$ & $0.129^{\mathrm{d}-\mathrm{g}}$ & $0.147^{\mathrm{d}-\mathrm{g}}$ \\
\hline $\mathrm{Zn}_{3.0} \mathrm{~B}_{1.0}$ & $22.1^{\mathrm{ab}}$ & $21.1^{a-d}$ & $0.130^{\mathrm{bcd}}$ & $0.143^{a b c}$ & $0.143^{\mathrm{cd}}$ & $0.174^{\mathrm{abc}}$ \\
\hline $\mathrm{Zn}_{3.0} \mathrm{~B}_{1.5}$ & $22.9^{\mathrm{ab}}$ & $22.4^{\mathrm{ab}}$ & $0.147^{a b}$ & $0.153^{\mathrm{ab}}$ & $0.164^{\mathrm{ab}}$ & $0.182^{\mathrm{ab}}$ \\
\hline $\mathrm{Zn}_{3.0} \mathrm{~B}_{2.0}$ & $24.4^{\mathrm{a}}$ & $23.7^{a}$ & $0.158^{a}$ & $0.163^{a}$ & $0.176^{a}$ & $0.190^{a}$ \\
\hline CV (\%) & 5.76 & 7.99 & 12.8 & 11.9 & 8.83 & 9.43 \\
\hline LSD (0.05) & 3.73 & 2.74 & 0.028 & 0.025 & 0.02 & 0.024 \\
\hline
\end{tabular}

Values within the same column with a common letter do not differ significantly $(P<0.05)$.

nodules per plant at 32 DAS ranged across the different treatments from 8.57 to 16.3 at Madaripur and 15.6 to 20.1 at Jashore. Number nodules per plant at 47 DAS varied from 23.6 to 32.7 at Madaripur and 29.9 to 40.5 at Jashore while at 62 DAS the number of nodules per plant also ranged from 30.6 to 46.8 at Madaripur and 41.0 to 49.8 at Jashore. Furthermore, at 77 DAS, it ranged across treatments from 19.8 to 26.1 at Madaripur and 25.7 to 33.7 at Jashore. The highest number of nodules per plant was recorded in $\mathrm{Zn}_{3.0} \mathrm{~B}_{2.0}$ treatment in all the nodule collection dates which showed significant variation with the others treatment except the collection date of 77 DAS. The minimum number of nodules per plant was recorded in control treatment $\left(\mathrm{Zn}_{0} \mathrm{~B}_{0}\right)$ in all nodule collection dates except the collection date of 77 DAS (Table 4).

\section{Effect of Zinc and Boron on protein content in seed and $\mathrm{Zn}$ and $\mathrm{B}$ uptake by fieldpea}

The highest protein content $(24.4 \%$ at Madaripur and $23.7 \%$ at Jessore) was recorded from the treatment $\mathrm{Zn}_{3.0} \mathrm{~B}_{2.0}$ which was significantly higher than others treatments at Jashore as well as Madaripur. The effect was consistence across the location. The lowest protein content in seed $(18.8 \%$ at Madaripur and $18.3 \%$ at Jashore) was recorded from the treatment $\mathrm{Zn}_{0} \mathrm{~B}_{0}$ (Table 5).

Different combinations of $\mathrm{Zn}$ and $\mathrm{B}$ showed significant effect on uptake of $\mathrm{Zn}$ and $\mathrm{B}$ by the fieldpea at Madaripur and Jashore (Table 5). The uptake of $\mathrm{Zn}$ by fieldpea under different combinations of $Z \mathrm{n}$ and $B$ ranged from $0.081 \mathrm{~kg}$ $\mathrm{Zn} \mathrm{ha}{ }^{-1}$ to $0.158 \mathrm{~kg} \mathrm{Zn} \mathrm{ha}^{-1}$ at Madaripur and from $0.082 \mathrm{~kg}$
$\mathrm{Zn} \mathrm{ha}{ }^{-1}$ to $0.163 \mathrm{~kg} \mathrm{Zn} \mathrm{ha}^{-1}$ at Jashore. The highest uptakes of $\mathrm{Zn}$ by fieldpea at both the locations were observed in treatment combination of $\mathrm{Zn}_{3.0} \mathrm{~B}_{2.0}$ which was significantly higher than others treatments but statistically at par with $\mathrm{Zn}_{3.0} \mathrm{~B}_{1.5}$ at Madaripur and with $\mathrm{Zn}_{3.0} \mathrm{~B}_{1.5}, \mathrm{Zn}_{3.0} \mathrm{~B}_{1.0}$ and $\mathrm{Zn} \mathrm{n}_{2.0} \mathrm{~B}_{2.0}$ at Jashore. The uptake of $\mathrm{B}$ by fieldpea due to different combinations of $\mathrm{Zn}$ and $\mathrm{B}$ varied from 0.097 to $0.176 \mathrm{~kg} \mathrm{~B} \mathrm{ha}^{-1}$ at Madaripur and 0.103 to $0.190 \mathrm{~kg} \mathrm{~B} \mathrm{ha}^{-1}$ at Jashore, respectively. The highest $B$ uptake was found in the treatment combination of $Z_{3.0} B_{2.0}$ which was significantly different with the other treatments, but statistically identical to $\mathrm{Zn}_{3.0} \mathrm{~B}_{1.5}$ at Madaripur and statistically alike to $Z_{3.0} B_{1.5}, Z_{3.0} B_{1.0}$ and $Z_{2.0} B_{2.0}$ at Jashore. However, the lowest uptakes of $\mathrm{Zn}$ and $\mathrm{B}$ by test crop were found in $\mathrm{Zn}_{0} \mathrm{~B}_{0}$ treatment (Table 5).

\section{Effect of $\mathrm{Zn}$ and $\mathrm{B}$ on postharvest soil properties}

Soil samples were collected from each plot for analysing different soil properties viz. soil $\mathrm{pH}$, organic matter, total $\mathrm{N}$ and available $\mathrm{P}, \mathrm{K}, \mathrm{S}, \mathrm{Ca}, \mathrm{Zn}$ and $\mathrm{B}$. Initially, the soil $\mathrm{pH}$ was 7.4 at Madaripur and 8.3 at Jashore, and the postharvest soil $\mathrm{pH}$ remained nearly unchanged. Comparing between the initial and post harvest soil properties - a minor fertility variation was found in post harvest soil in combine applications of different rates of $\mathrm{Zn}$ and $B$ at Madaripur and Jashore. The combine application of $\mathrm{Zn}$ and $\mathrm{B}$ tended to maintain the initial fertility or increased slightly. The treatment $Z_{3.0} \mathrm{~B}_{2.0}$ followed by $\mathrm{Zn}_{3.0} \mathrm{~B}_{1.5}$ showed encouraging results of organic matter, $\mathrm{N}$, $\mathrm{P}, \mathrm{S}, \mathrm{Ca}, \mathrm{Zn}$ and $\mathrm{B}$ at both the locations. Potassium slightly 
Table 6. Effect of $\mathrm{Zn}$ and $\mathrm{B}$ on postharvest soil $\mathrm{pH}$ and the status of different nutrients at Madaripur and Jashore (mean of two years) with reference to initial soil.

\begin{tabular}{|c|c|c|c|c|c|c|c|c|c|}
\hline Treatments & $\mathrm{pH}$ & $\begin{array}{l}\text { OM } \\
(\%)\end{array}$ & $\begin{array}{c}\text { Total N } \\
(\%)\end{array}$ & $\begin{array}{c}\mathrm{Ca} \text { (meq. } \\
\left.100 \mathrm{~g}^{-1}\right)\end{array}$ & $\begin{array}{l}\mathrm{K} \text { (meq. } \\
\left.100 \mathrm{~g}^{-1}\right)\end{array}$ & $\begin{array}{c}P \\
\left(\mu g^{-1}\right)\end{array}$ & $\begin{array}{c}S \\
\left(\mu g g^{-1}\right)\end{array}$ & $\begin{array}{c}\mathrm{Zn} \\
\left(\mu \mathrm{g} \mathrm{g}^{-1}\right)\end{array}$ & $\begin{array}{c}\text { B } \\
\left(\mu \mathrm{g} \mathrm{g}^{-1}\right)\end{array}$ \\
\hline \multicolumn{10}{|l|}{ Madaripur } \\
\hline Initial & 7.4 & 1.42 & 0.063 & 12.8 & 0.15 & 14.0 & 16.0 & 1.18 & 0.15 \\
\hline $\mathrm{Zn}_{0} \mathrm{~B}_{0}$ & 7.3 & 1.41 & 0.062 & 12.6 & 0.13 & 13.9 & 16.1 & 1.17 & 0.13 \\
\hline $\mathrm{Zn}_{0} \mathrm{~B}_{1.0}$ & 7.4 & 1.43 & 0.064 & 12.7 & 0.14 & 14.8 & 16.8 & 1.17 & 0.16 \\
\hline $\mathrm{Zn}_{0} \mathrm{~B}_{1.5}$ & 7.4 & 1.45 & 0.065 & 12.9 & 0.14 & 15.0 & 17.0 & 1.19 & 0.17 \\
\hline $\mathrm{Zn}_{0} \mathrm{~B}_{2.0}$ & 7.3 & 1.50 & 0.067 & 12.8 & 0.13 & 15.1 & 17.3 & 1.17 & 0.17 \\
\hline $\mathrm{Zn}_{1.0} \mathrm{~B}_{0}$ & 7.5 & 1.47 & 0.066 & 12.5 & 0.15 & 14.5 & 17.0 & 1.18 & 0.15 \\
\hline $\mathrm{Zn}_{1.0} \mathrm{~B}_{1.0}$ & 7.4 & 1.48 & 0.067 & 13.1 & 0.13 & 15.0 & 16.5 & 1.19 & 0.16 \\
\hline $\mathrm{Zn}_{1.0} \mathrm{~B}_{1.5}$ & 7.3 & 1.49 & 0.067 & 13.4 & 0.13 & 15.2 & 16.0 & 1.20 & 0.17 \\
\hline $\mathrm{Zn}_{1.0} \mathrm{~B}_{2.0}$ & 7.4 & 1.48 & 0.066 & 13.3 & 0.14 & 15.3 & 16.1 & 1.21 & 0.18 \\
\hline $\mathrm{Zn}_{2.0} \mathrm{~B}_{0}$ & 7.4 & 1.47 & 0.066 & 12.7 & 0.15 & 14.9 & 15.9 & 1.20 & 0.14 \\
\hline $\mathrm{Zn}_{2.0} \mathrm{~B}_{1.0}$ & 7.3 & 1.50 & 0.067 & 13.2 & 0.13 & 15.1 & 16.2 & 1.23 & 0.16 \\
\hline $\mathrm{Zn}_{2.0} \mathrm{~B}_{1.5}$ & 7.4 & 1.52 & 0.068 & 13.5 & 0.12 & 16.0 & 16.5 & 1.24 & 0.17 \\
\hline $\mathrm{Zn}_{2.0} \mathrm{~B}_{2.0}$ & 7.3 & 1.53 & 0.069 & 13.6 & 0.13 & 15.8 & 17.0 & 1.25 & 0.18 \\
\hline $\mathrm{Zn}_{3.0} \mathrm{~B}_{0}$ & 7.4 & 1.51 & 0.067 & 12.9 & 0.14 & 14.7 & 16.1 & 1.25 & 0.15 \\
\hline $\mathrm{Zn}_{3.0} \mathrm{~B}_{1.0}$ & 7.5 & 1.53 & 0.069 & 13.5 & 0.13 & 16.1 & 17.1 & 1.24 & 0.16 \\
\hline $\mathrm{Zn}_{3.0} \mathrm{~B}_{1.5}$ & 7.4 & 1.54 & 0.070 & 13.4 & 0.12 & 15.9 & 17.2 & 1.26 & 0.17 \\
\hline $\mathrm{Zn}_{3.0} \mathrm{~B}_{2.0}$ & 7.5 & 1.54 & 0.071 & 13.6 & 0.12 & 16.1 & 17.2 & 1.26 & 0.18 \\
\hline \multicolumn{10}{|l|}{ Jessore } \\
\hline Initial & 8.3 & 1.64 & 0.072 & 16.7 & 0.16 & 14.6 & 16.5 & 1.10 & 0.16 \\
\hline $\mathrm{Zn}_{0} \mathrm{~B}_{0}$ & 8.2 & 1.65 & 0.073 & 16.3 & 0.13 & 14.0 & 16.4 & 1.07 & 0.15 \\
\hline $\mathrm{Zn}_{0} \mathrm{~B}_{1.0}$ & 8.3 & 1.69 & 0.074 & 16.8 & 0.15 & 14.8 & 17.0 & 1.06 & 0.17 \\
\hline $\mathrm{Zn}_{0} \mathrm{~B}_{1.5}$ & 8.2 & 1.70 & 0.074 & 16.6 & 0.15 & 15.0 & 17.1 & 1.05 & 0.20 \\
\hline $\mathrm{Zn}_{0} \mathrm{~B}_{2.0}$ & 8.2 & 1.70 & 0.075 & 16.7 & 0.15 & 15.3 & 17.1 & 1.06 & 0.22 \\
\hline $\mathrm{Zn}_{1.0} \mathrm{~B}_{0}$ & 8.2 & 1.72 & 0.076 & 16.8 & 0.15 & 15.4 & 17.0 & 1.13 & 0.16 \\
\hline $\mathrm{Zn}_{1.0} \mathrm{~B}_{1.0}$ & 8.3 & 1.71 & 0.074 & 16.9 & 0.14 & 15.4 & 17.2 & 1.14 & 0.19 \\
\hline $\mathrm{Zn}_{1.0} \mathrm{~B}_{1.5}$ & 8.3 & 1.72 & 0.075 & 17.0 & 0.14 & 15.3 & 17.3 & 1.15 & 0.22 \\
\hline $\mathrm{Zn}_{1.0} \mathrm{~B}_{2.0}$ & 8.2 & 1.73 & 0.076 & 16.8 & 0.14 & 15.4 & 17.3 & 1.15 & 0.24 \\
\hline $\mathrm{Zn}_{2.0} \mathrm{~B}_{0}$ & 8.2 & 1.72 & 0.075 & 16.7 & 0.15 & 15.4 & 16.8 & 1.18 & 0.16 \\
\hline $\mathrm{Zn}_{2.0} \mathrm{~B}_{1.0}$ & 8.3 & 1.73 & 0.077 & 17.1 & 0.13 & 16.0 & 17.0 & 1.18 & 0.18 \\
\hline $\mathrm{Zn}_{2.0} \mathrm{~B}_{1.5}$ & 8.3 & 1.74 & 0.077 & 17.2 & 0.13 & 16.1 & 17.1 & 1.19 & 0.23 \\
\hline $\mathrm{Zn}_{2.0} \mathrm{~B}_{2.0}$ & 8.3 & 1.75 & 0.078 & 17.1 & 0.13 & 16.3 & 17.3 & 1.19 & 0.26 \\
\hline $\mathrm{Zn}_{3.0 \mathrm{~B}_{0}}$ & 8.2 & 1.74 & 0.077 & 16.9 & 0.14 & 16.1 & 17.0 & 1.23 & 0.17 \\
\hline $\mathrm{Zn}_{3.0} \mathrm{~B}_{1.0}$ & 8.2 & 1.75 & 0.078 & 17.3 & 0.13 & 16.5 & 17.3 & 1.26 & 0.22 \\
\hline $\mathrm{Zn}_{3.0} \mathrm{~B}_{1.5}$ & 8.3 & 1.76 & 0.079 & 17.6 & 0.13 & 16.4 & 17.4 & 1.27 & 0.25 \\
\hline $\mathrm{Zn}_{3.0} \mathrm{~B}_{2.0}$ & 8.2 & 1.77 & 0.080 & 17.7 & 0.13 & 16.5 & 17.4 & 1.27 & 0.27 \\
\hline
\end{tabular}

decreased in all treatments from the initial $\mathrm{K}$ status (Table $6)$. The soil fertility status of Madaripur was comeratively inferior to the fertility status of Jashore (Table 6).

\section{DISCUSSION}

Pulse crops are usually grown under rain-fed condition without proper nutrient management practices espacially micronutrient. Pulses crop needs timely application of optimum micronutrient for plant growth, quality seed and high seed yield. Therefore, the different combination of $\mathrm{Zn}$ and $B$ application on fieldpea in calcareous soils were compared. The combine effects of $\mathrm{Zn}$ and B contributed significantly to increase seed and stover yields of fieldpea. Micronutrient ( $\mathrm{Zn}$ and $\mathrm{B}$ ) may assist in translocation of photosynthates resulting better pod formation as well as 


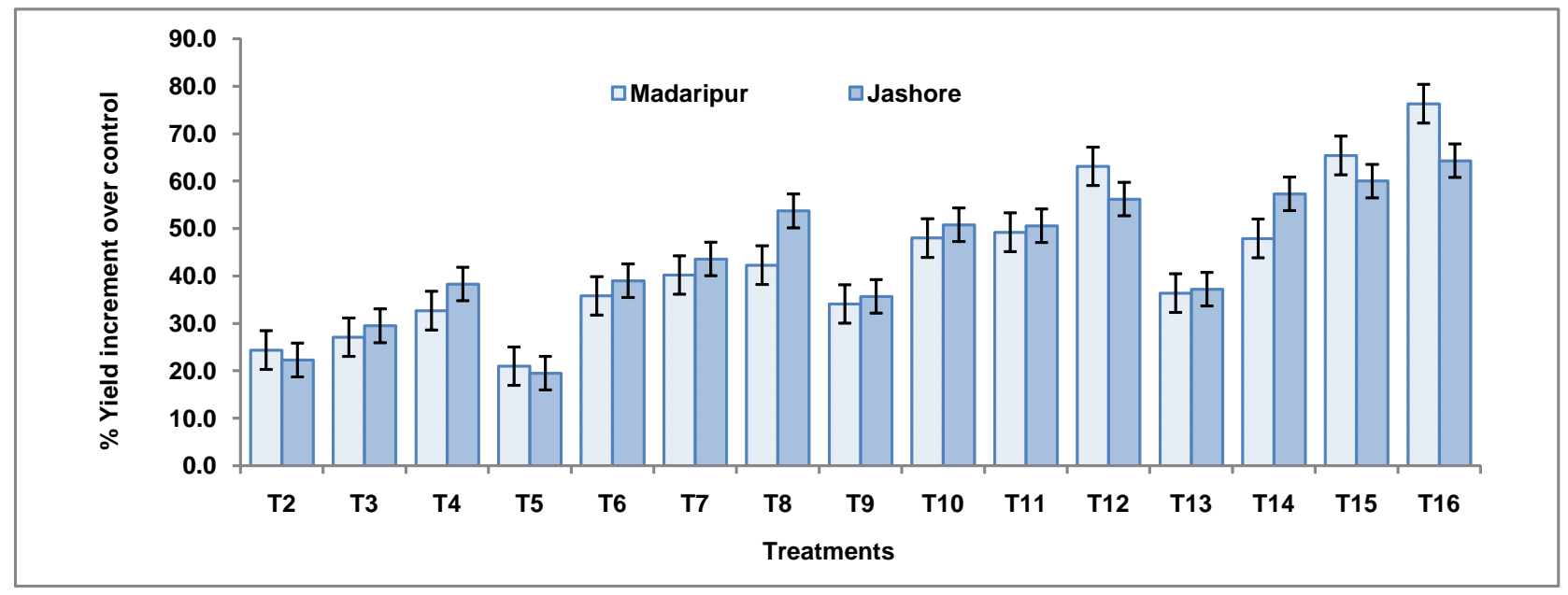

Figure 3. Combine effect of zinc and boron on \% seed yield increment of fieldpea over control at Madaripur and Jashore. Note: Errors bars represent the SEM. Treatment: $\mathrm{T}_{2}=\mathrm{Zn}_{0} \mathrm{~B}_{1.0} ; \mathrm{T}_{3}=Z \mathrm{n}_{0} \mathrm{~B}_{1.5} ; \mathrm{T}_{4}=\mathrm{Zn}_{0} \mathrm{~B}_{2.0} ; \mathrm{T}_{5}=\mathrm{Zn}_{1.0} \mathrm{~B}_{0} ; \mathrm{T}_{6}=\mathrm{Zn}_{1.0} \mathrm{~B}_{1.0} ; \mathrm{T}_{7}=$ $\mathrm{Zn}_{1.0} \mathrm{~B}_{1.5} ; \mathrm{T}_{8}=\mathrm{Zn}_{1.0} \mathrm{~B}_{2.0} ; \mathrm{T}_{9}=\mathrm{Zn}_{2.0} \mathrm{~B}_{0} ; \mathrm{T}_{10}=\mathrm{Zn}_{2.0} \mathrm{~B}_{1.0} ; \mathrm{T}_{11}=\mathrm{Zn}_{2.0} \mathrm{~B}_{1.5} ; \mathrm{T}_{12}=\mathrm{Zn}_{2.0} \mathrm{~B}_{2.0} ; \mathrm{T}_{13}=\mathrm{Zn}_{3.0} \mathrm{~B}_{0} ; \mathrm{T}_{14}=\mathrm{Zn}_{3.0} \mathrm{~B}_{1.0} ; \mathrm{T}_{15}=\mathrm{Zn}_{3.0} \mathrm{~B}_{1.5}$; and $\mathrm{T}_{16}=\mathrm{Zn}_{3.0} \mathrm{~B}_{2.0}$.
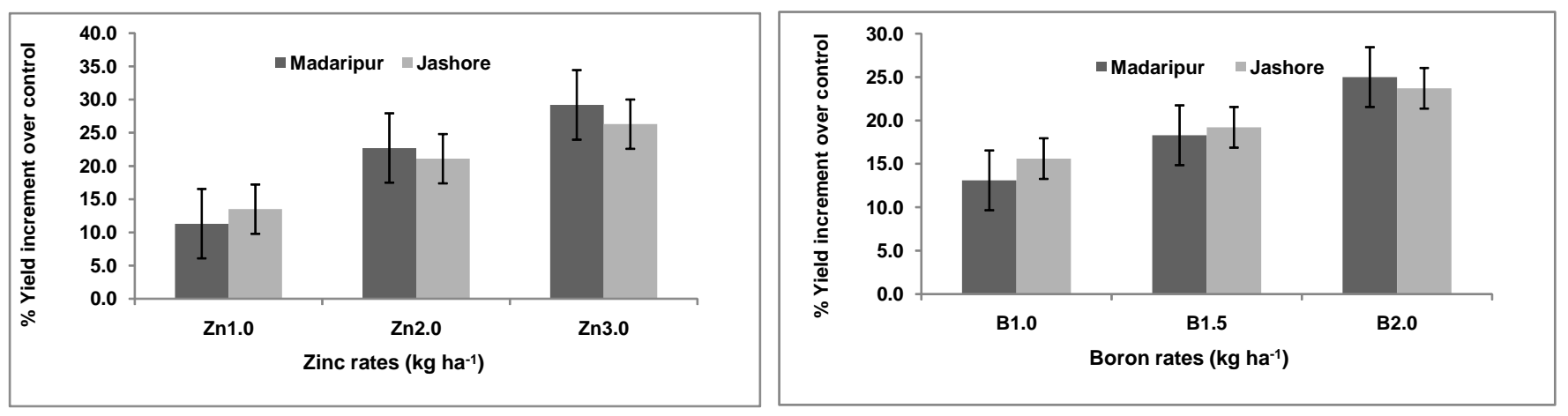

Figure 4. Single effect of zinc and boron on \% seed yield increment of fieldpea over control at Madaripur and Jashore. Note: Errors bars represent the SEM.

quality seeds which ultimately increase the seed yield. The results are in agreement with the findings of Kumar (2011). The experimental soils were deficient of organic matter, nitrogen, available potassium, zinc and boron thus crop responded positively to application of fertilizer and soil fertility level was improved through addition of $N, P, K, S$, $\mathrm{Zn}$ and $\mathrm{B}$. The same findings were observed by Agrawal and Sharma (2005) and Mevada et al. (2005). Combine application of $\mathrm{Zn}$ and $\mathrm{B}$ were found effective in producing more seed and stover yield of fieldpea over control. Hence, micronutrient ( $\mathrm{Zn}$ and $\mathrm{B}$ ) might have enhanced the existence of the soil fertility and multiplication of soil microorganism, resulting in more nitrogen fixation, better transport of sugar and higher uptake and assimilation of available nutrients by the plants. Similar observations have been reported by Kumar et al. (2009) and Valenciano et al. (2010). Integrated use of Zinc and Boron was found more effective over their single application in terms of seed and stover yield of fieldpea. These explanations could be well understood when seed yield increment (\%) over control by different treatments are compared. Integrated use of $\mathrm{Zn}$ and B contributed 21.0 to $76.3 \%$ yield increment over control at Madaripur and 19.5 to $64.3 \%$ at Jashore (Figure 3 ). But single application of zinc contributed only 11.3 to $29.2 \%$ yield increment over control at Madaripur and 13.5 to $26.3 \%$ at Jashore. Similarly, single application of boron produced 13.1 to $25.0 \%$ yield increment over control at Madaripur and 15.6 to $23.7 \%$ yield increment over control at Jashore (Figure 4). Thus, integrated use of Boron and Zinc are effective in increasing the yield of fieldpea in the study areas.

Effective nodulation of fieldpea was ensured due to combine application of $\mathrm{Zn}$ and $\mathrm{B}$ or single application of $\mathrm{Zn} / \mathrm{B}$ along with macro ( $\mathrm{N}, \mathrm{P}, \mathrm{K}$ and $\mathrm{S}$ ) nutrients. Different combinations of $\mathrm{Zn}$ and $\mathrm{B}$ demonstrated that the number of nodules per plant was significantly increased in all nodule collection dates in both locations of Madaripur and Jashore. From the results of different dates, nodule 
formation was less in 32 DAS and in 77 DAS but, the formation was maximum between 47 and 62 DAS. It seems that the highest numbers of nodules formation occurred during early to mid flowering stage. After flowering, nodule efficiency might be reduced and began to shut down. This finding is supported by Kevin (2006). Noor and Hossain (2007) reported that adequate Boron ensured effective nodulation and nitrogen fixation in legumes. Zinc plays a vital role in metabolism and is involved in $\mathrm{N}$-fixation through nodule formation (Patel et al. 2011). It was observed also from the study that combined applications of Boron and Zinc were more effective for nodule formation than their single application.

The protein percentage in fieldpea was affected significantly due to combine or single application of Zinc and Boron including macro (N, P, K and S) nutrients. Results of this study reveal that the different levels of $\mathrm{Zn}$ and $B$ application contributed positively in increasing the protein content in fieldpea seed. Micronutrient ( $\mathrm{Zn}$ and B) might be play a key role in protein synthesis. Mali et al. (2003) found that proper doses of zinc application might enhanced the synthesis of carbohydrates and protein and their transport to the site of seed formation. Boron influences the absorption of $\mathrm{N}, \mathrm{P}, \mathrm{K}$ and it has positive role on protein synthesis (Chatterjee and Subhendu 2015). The present study documented that the combine application of $\mathrm{Zn}$ and $\mathrm{B}$ influence significantly the uptakes of $\mathrm{Zn}$ and $\mathrm{B}$ by fieldpea. In this research, the Zinc and Boron uptake were increased in crop due to increasing the rates of $Z n$ and $B$ and that was due to deficiency of micronutrients in experimental field. The maximum $\mathrm{Zn}$ and $\mathrm{B}$ uptakes were achieved by seed and stover of fieldpea in associations with higher applied of nutrients including $\mathrm{Zn}$ and $\mathrm{B}$ which contributed to maximum seed and stover yield. Integrated use of micronutrients along with macro might help in higher acquisition of atmospheric nitrogen in nodule by symbiotic microbes making it available to the pea plant (Kumar et al., 2009).

The observation of soil fertility indicated that fieldpea might help to enrich and conserve the soil quality for enhancing the yield of next crop. Similar observation was made by Musinguzi et al. (2010). Pulses are able to convert atmospheric nitrogen into nitrogen compounds that can be used by plants, while also improving soil fertility (Nulik et al., 2013). The presence of pulses in agroecosystems helps to maintainvital microbial biomass and activity in the soil, in that way nourishing those organisms that are responsible for promoting soil structure and nutrient availability (Blanchart et al., 2005).

The above results and explanations further highlighted the requirement for targeted soil fertility management through micronutrient application in order to monitor the dose to achieve sustainable crop (fieldpea) production.

\section{Conclusions}

Combine application of Zinc at $3 \mathrm{~kg} \mathrm{ha}^{-1}$ and Boron at $2 \mathrm{~kg}$ $\mathrm{ha}^{-1}$ significantly increased the seed yield of fieldpea. The maximum nodulation in root and protein percentage in seed was found in the treatment combination of $\mathrm{Zn}_{3.0} \mathrm{~B}_{2.0}$ followed by $Z_{3.0} B_{1.5}$. Similarly, $Z n$ and $B$ uptake was also higher in the treatment of $Z_{3.0} B_{2.0}$ followed by $Z_{3.0} B_{1.5}$ Thus, the application of $\mathrm{Zn}$ and $\mathrm{B}$ at the rate $3 \mathrm{~kg} \mathrm{Zn} \mathrm{ha-1}$ and $2 \mathrm{~kg} \mathrm{~B} \mathrm{ha}^{-1}$ influenced significantly on fieldpea yield and other parameters. Combine application of $\mathrm{Zn}$ and $\mathrm{B}$ was more effective than their single application. The combine application of $\mathrm{Zn}$ and $\mathrm{B}$ improved the soil organic matter, N, P, S, Zn and B in both the locations. So, the combination of $Z n_{3.0} B_{2.0}$ and $Z n_{3.0} B_{1.5}$ along with $N_{12} P_{22} K_{30}$ $\mathrm{S}_{10} \mathrm{~kg} \mathrm{ha}^{-1}$ might be recommended for maximum yield production of fieldpea in calcareous soils. Therefore, attention should be given to improve fieldpea production through micronutrient management.

\section{CONFLICT OF INTEREST STATEMENT}

The authors declare that there are no conflicts of interest.

\section{ACKNOWLEDGEMENT}

This research work was conducted by the financial support of Pulses Research Centre in Bangladesh Agricultural Research Institute. The authors would like to thank Dr. M.A. Quyayyum for giving valuable comments and suggestions on earlier drafts of the manuscript. Authors are grateful to the Head of Soil Science Division for providing laboratory facility to analyze the plant and soil samples.

\section{REFERENCES}

Agrawal, M. M., \& Sharma, C. P. (2005). Effect of sulphur and molybdenum with Rhizobium and PSB on yield and nutrient uptake in chick pea. Farm Science Journal, 15, 20-2.

Ali, A., Ahmad, B., Hussain, I., Ali, A., \& Shah, F. A (2017). Effect of phosphorus and zinc on yield of lentil. Pure and Applied Biology, 6(4), 1397-1402.

Bangladesh Bureau of Statistics (BBS). (2016). Yearbook of Agricultural Statistics-2015, 27th series, Statistics and Information Division, Ministry of Planning, Government of the people's Republic of Bangladesh. Available at www.bbs.gov.bd

Blanchart, E., Villenave, C., Viallatoux, A., Barthès, B., Girardin, C., Azontonde, A., \& Feller, C. (2005). Long-term effect of a legume cover crop (Mucuna pruriens var. utilis) on the communities of soil macrofauna and nematofauna under maize cultivation, in southern Benin. European Journal of Soil Biology, 42 (S1), 136-144.

Bremner, J. M., \& Mulvaney, C.S. (1982). Total nitrogen. In A. L. Page, R. H. Miller, D. R. Keeney (Eds.), Methods of Soil Analysis (Part 2, 2nd ed., Pp. 599-622). American Society of Agronomy, Madison, USA.

Chatterjee, R., \& Subhendu, B. (2015). Effect of boron, molybdenum and biofertilizers on growth and yield of cowpea 
(Vigna unguiculata L. Walp.) in acid soil of eastern Himalayan region. Journal of the Saudi Society of Agricultural Sciences, 16, 332-336.

Erman, M., Yildirim, B., Necat, T., \& Fatih, C. (2009). Effect of phosphorus application and Rhizobium inoculation on the yield, noducation and nutrient uptake in field pea (Pisum sativum sp. arvense L.). Journal of Animal and Veterinary Advances, 8(2),301-304.

Fertilizer Recommendation Guide (FRG). (2012). Fertilizer Recommendation Guide, Published by Bangladesh Agricultural Research Council, Dhaka, Bangladesh.

Food and Agriculture Organization (FAO) (2018). Analysis of Protein.Food and agriculture Origination publication, FAO Food and Nutrition Paper 14/7. Centre for Food Safety, UN. Available at http://www.cfs.gov.hk/english/programme/programme_nifl/file s/Analysis_of_Protein.pdf. Accessed on 08 January 2018.

Fox, R.vL., Olsen, R. A., \& Rhoades, H. F. (1964). Evaluating the sulphur status of soil by plant and soil test. Soil Science Society of America Journal, 28(2), 243-246.

Gupta, P. K. (2004). Soil, Plant, Water and Fertilizer Analysis. Department of Agricultural Chemistry and Soil Science, MaharanaPratap University of Agriculture \& Technology, Rajasthan, India. Pp. 168-170.

Jackson, M. L. (1973). Soil Chemical Analysis.Prentice Hall of India Private Limited, New Delhi.pp 498.

Jahiruddin, M. (2015). Zinc and Boron deficiency in crops and their management in Bangladesh. Department of Soil Science, Bangladesh Agricultural University, Mymensingh, Pp. 1-27.

Karan, D., Singh, S. B., \& Ramkewal, (2014). Effect of zinc and boron application on yield of lentil and nutrient balance in the soil under Indo-Gangetic plain zones. Journal of AgriSearch, 1(4), 206-209.

Kevin, Z. (2006). Nodulation and nitrogen fixation field guide for the assessment of pulse crops. Edited by Kevin Zaychuk, 20/20 Seed Labs Inc-December, 2006. Available at http://www.agr.gov.sk.ca/docs/production/inoculationpulses.a $\mathrm{sp}$

Kumar, J. (2011). Effect of phosphorus and sulphur application on performance of vegetable pea (Pisum sativum L.) cv. Pant Matar-2. Legume Research, 34(4), 292-5.

Kumar, R. P., Singh, O. N., Singh, Y., Dwivedi, S., \& Singh, J. P., (2009). Effect of Integrated nutrient management on growth, yield nutrient uptake and economics of French bean (Phaseolus vulgaris). Indian Journal of Agricultural Sciences, 9(2), 122-8.

Kumar, S., \& Singh, M. (2009). 25 Years of Pulses Research at IIPR, 1984-2009 (Editors Shiv Kumar and Mohan Singh), Published by: Indian Institute of Pulses Research, Kanpur 208024, India

Lindsay, W. L., \& Norvell, W. A. (1978). Development of DTPA soil test for zinc, iron, manganese and copper. Soil science Society of American Journal, 42, 421-8.

Mali, G. C., Sharma, N. N., Acharya, H. K., Gupta, S. K., \& Gupta, P. K. (2003). Response of pigeon pea to $S$ and $Z n$ fertilization on vertisols in south-eastern plain of Rajasthan. Advances in Arid Legumes Research, Pp. 267-271. Indian Arid Legumes Society, Scientific Publishers (India), Jodhpur.

Mevada, K. D., Patel, J. J., \& Patel, K. P. (2005). Effect of micronutrients on yield of urdbean. Indian Journal of Pulses Research, 18, 214-6.

Musinguzi, P., Tenywa, J. S., \& Bekunda, M. A. (2010). Strategic nutrient management of field pea In Southwestern Uganda. African Journal of Food Agriculture Nutrition and Development
10(6), 2695-2706.

Nelson, D. W., \& Sommers, L. E. (1982). Total carbon, organic carbon and organic matter. In Page, A. L., Miller, R. H., \& Keeney, D. R. (Eds.), Methods of Soil Analysis (Part 2, 2nd ed., Pp. 539-580). American Society of Agronomy, Madison, USA.

Noor, S. S., \& Hossain, M. A. (2007). Effects of boron and molybdenum on the yield of chickpea. Journal of Agricultural and Rural Development, 5 (1\&2), 17-24.

Nulik, J., Dalgliesh, N., Cox, K., \& Gabb, S. (2013). Integrating herbaceous legumes into crop and livestock systems in eastern Indonesia. Canberra, Australia: Australian Centre for International Agricultural Research.

Oelke, E. A., Oplinger, E. S., Hanson, C. V., Davis, D. W., Putnam, D. H., Fuller, E. I., \& Rosen, C. J. (1991). Field peaWisconsin Corn Agronomy. University of Wisconsin-Madison, WI $53706 . \quad$ Available at http://corn.agronomy.wisc.edu/CropsPea.aspx

Olsen, S., \& Sommer, L. E. (1982). Phosphorus. In Page, A. L., Miller, R. H., \& D. R. Keeney (Eds.), Methods of Soil Analysis (Part 2, 2nd ed., Pp. 403-427). American Society of Agronomy, Madison, USA.

Page, A. L., Miller, R. H., \& Keeney, D. R. (Eds.). (1982). Agronomy Series 9 ASA, SSSA.Methods of Soil Analysis (Part 2, 2nd ed., Pp. 403-427). American Society of Agronomy, Madison, USA.

Patel, M. M., Patel, I. C., Patel, R. I., \& Acharya, S. (2011). Effect of Zinc and Iron on yield and yield attributes of rainfed cowpea (Vigna unguiculata L. Walp). Annals of Arid Zone, 50(1), 1719.

Persson, J-A., Wennerholm, M., \& O'Halloram, S. (2008). Handbook for Kjeldahl Digestion. Published by FOSS, DK3400 Hilleroed, Denmark. ISBN 91-630-3471-9.

Piper, C. S. (1966). Soil and Plant Analysis. Adelaide University Press, Australia.

Quddus, M. A., Mian, M. A., Naser, H. M., Hossain, M. A., \& Sultana, S. (2017). Maximizing Yields, Nutrient Uptake and Balance for Mustard-Mungbean-T. Aman Rice Cropping Systems through Nutrient Management Practices in Calcareous Soils. Journal of Agricultural Science, 9(9), 210.

Quddus, M. A., Naser, H. M., Hossain, M. A., \& AbulHossain, M. (2014). Effect of Zinc and Boron on yield and yield contributing characters of lentil in low Ganges river floodplain soil at madaripur, bangladesh. Bangladesh Journal of Agricultural Research, 39(4), 591-603.

Quddus, M. A., Rashid, M. H., Hossain, M. A., \& Naser, H. M. (2011). Effect of zinc and boron on yield and yield contributing characters of mungbean in low Ganges river floodplain soil at madaripur, Bangladesh. Bangladesh Journal of Agricultural Research, 36(1), 75-85.

Rashid, M. M. (2001). Agroecological characteristics of Bangladesh.In: WadudMian M. A., Maniruzzaman, F. M., Sattar, M. A., Aziz Miah, M. A., Paul S. K., \& Haque K. R. (eds.) Agricultural Research in Bangladesh in the 20th Century. Bangladesh Agricultural Research Council \& Bangladesh Academy of Agriculture, Dhaka. Pp.37-42.

Singh, A. K., Khan, M. A., \& Srivastava, A. (2014). Effect of boron and molybdenum application on seed yield of mungbean. Asian Journal of Biolological Science, 9(2) 169-172.

Singh, D. K., Singh, A. K., Sanjay, K. S., Mandhata, S., \& Srivastava, O. P. (2015). Effect of balanced nutrition on yield and nutrient uptake of pea (Pisum sativum L.) under IndoGangetic plains of India. The Bioscan, 10(3), 1245-1249.

Statistix 10. (1985). An Anlytical Software, Po Box 12185, Tallahassee, FL 32317, Copy right (C) 1985-2013. 
Subasinghe, S., Dayatilake, G. A., \& Senaratne, R. (2003). Effect of $\mathrm{B}, \mathrm{Co}$ and Mo on nodulation, growth and yield of cowpea (Vigna unguiculata) Tropical Agricultural Research, Ext.6, 108112.
Valenciano, J. B., Bato, J. A., \& Marcelo, V. (2010). Response of chickpea (Cicer arietinum L.) yield to Zinc, boron and molybdenum application under pot conditions. Spanish Journal of Agricultural Research, 3, 797-807. 